\title{
Comparing FPGA vs. Custom CMOS and the Impact on Processor Microarchitecture
}

\author{
Henry Wong \\ Department of Electrical and \\ Computer Engineering \\ University of Toronto \\ henry@eecg.utoronto.ca
}

\author{
Vaughn Betz \\ Altera Corp. \\ vbetz@altera.com
}

\author{
Jonathan Rose \\ Department of Electrical and \\ Computer Engineering \\ University of Toronto \\ jayar@eecg.utoronto.ca
}

\begin{abstract}
As soft processors are increasingly used in diverse applications, there is a need to evolve their microarchitectures in a way that suits the FPGA implementation substrate. This paper compares the delay and area of a comprehensive set of processor building block circuits when implemented on custom CMOS and FPGA substrates. We then use the results of these comparisons to infer how the microarchitecture of soft processors on FPGAs should be different from hard processors on custom CMOS.

We find that the ratios of the area required by an FPGA to that of custom CMOS for different building blocks varies significantly more than the speed ratios. As area is often a key design constraint in FPGA circuits, area ratios have the most impact on microarchitecture choices. Complete processor cores have area ratios of $17-27 \times$ and delay ratios of $18-26 \times$. Building blocks that have dedicated hardware support on FPGAs such as SRAMs, adders, and multipliers are particularly area-efficient $(2-7 \times$ area ratio), while multiplexers and CAMs are particularly area-inefficient $(>100 \times$ area ratio), leading to cheaper ALUs, larger caches of low associativity, and more expensive bypass networks than on similar hard processors. We also find that a low delay ratio for pipeline latches $(12-19 \times)$ suggests soft processors should have pipeline depths $20 \%$ greater than hard processors of similar complexity.
\end{abstract}

\section{Categories and Subject Descriptors \\ B.7.1 [Integrated Circuits]: Types and Design Styles}

\section{General Terms}

Design, Measurement

\section{Keywords}

FPGA, CMOS, Area, Delay, Soft Processor

\section{INTRODUCTION}

Custom CMOS silicon logic processes, standard cell ASICs, and FPGAs are commonly-used substrates for implementing

Permission to make digital or hard copies of all or part of this work for personal or classroom use is granted without fee provided that copies are not made or distributed for profit or commercial advantage and that copies bear this notice and the full citation on the first page. To copy otherwise, to republish, to post on servers or to redistribute to lists, requires prior specific permission and/or a fee.

FPGA'11, February 27-March 1, 2011, Monterey, California, USA.

Copyright 2011 ACM 978-1-4503-0554-9/11/02 ...\$10.00. digital circuits, with circuits implemented on each one having different characteristics such as delay, area, and power. In this paper, we compare custom CMOS and FPGA substrates for implementing processors and explore the microarchitecture trade-off space of soft processors in light of the differences.

FPGA soft processors have mostly employed single-issue in-order microarchitectures due to the limited area budget available on FPGAs [1-3]. We believe that future soft processor microarchitecture design decisions can benefit from a quantitative understanding of the differences between custom CMOS and FPGA substrates. Another reason to revisit the soft processor microarchitecture trade-off space is that the increased capacity of modern FPGAs can accommodate much larger soft processors if it results in a performance benefit.

Previous studies have measured the relative delay and area of FPGA, standard cell, and custom CMOS substrates as an average across a large set of benchmark circuits $[4,5]$. While these earlier results are useful in determining an estimate of the size and speed of circuit designs that can be implemented on FPGAs, we need to compare the relative performance of various types of "building block" circuits in order to have enough detail to guide microarchitecture design decisions.

This paper makes two contributions:

- We compare delay and area between FPGA and custom CMOS implementations of a set of building block circuits. While prior work gave results for complete systems, we show specific strengths and weaknesses of the FPGA substrate for the different building blocks.

- Based on the delay and area ratios of building block circuits on FPGAs vs. custom CMOS, we discuss how processor microarchitecture design trade-offs change on an FPGA substrate and the suitability of existing processor microarchitecture techniques when used on FPGAs.

We begin with background in Section 2 and methodology in Section 3. We then present our comparison results and their impact on microarchitecture in Sections 4 and 5, and conclude in Section 6.

\section{BACKGROUND}

\subsection{Technology Impact on Microarchitecture}

Studies on how process technology trends impact microarchitecture are essential for designing effective microarchitectures that take advantage of ever-changing manufacturing 
processes. Issues currently facing CMOS technology include poor wire delay scaling, high power consumption, and more recently, process variation. Microarchitectural techniques that respond to these challenges include clustered processor microarchitectures and chip multiprocessors $[6,7]$.

FPGA-implemented circuits face a very different set of constraints. Power consumption is not currently the dominant design constraint due to lower clock speeds, while area is often the primary constraint due to high area overheads of FPGA circuits. Characteristics such as inefficient multiplexers and the need to map RAM structures into FPGA hard SRAM blocks are known and generally adjusted for by modifying circuit-level, but not microarchitecture-level, design [8-11].

\subsection{Measurement of FPGAs}

Kuon and Rose have measured the area, delay, and power overheads of FPGAs compared to a standard cell ASIC flow on $90 \mathrm{~nm}$ processes [4] using a benchmark set of complete circuits to measure the overall impact of using FPGAs compared to ASICs and the effect of FPGA hard blocks. They found that circuits implemented on FPGAs consumed $35 \times$ more area than on standard cell ASIC for circuits that did not use hard memory or multiplier blocks, to a low of $18 \times$ for those that used both types. Minimum cycle time of the FPGA circuits were not significantly affected by hard blocks, ranging from 3.0 to $3.5 \times$ greater than ASIC. Chinnery and Keutzer [5] made similar comparisons between standard cell and custom CMOS and reported a delay ratio of 3 to $8 \times$. Combined, these reports suggest that the delay of circuits implemented on an FPGA would be 9 to $28 \times$ greater than on custom CMOS. However, data for full circuits are insufficiently detailed to guide microarchitecture-level decisions.

\section{METHODOLOGY}

We seek to measure the delay and area of FPGA building block circuits and compare them against their custom CMOS counterparts, resulting in area ratios and delay ratios. We define these ratios to be the area or delay of an FPGA circuit divided by the area or delay of the custom CMOS circuit. A higher ratio means the FPGA implementation has more overhead. We compare several complete processor cores and a set of building block circuits against their custom CMOS implementations, then observe which types of building block circuits have particularly high or low overhead on FPGA.

As we do not have the expertise to implement highlyoptimized custom CMOS circuits, our building block circuit comparisons use data from custom CMOS implementations found in the literature. The set of building block circuits is generic enough so that there are sufficient published delay and area data. We focus mainly on custom designs built in $65 \mathrm{~nm}$ processes, because it is the most recent process where design examples are readily available in the literature, and compare them to the Altera Stratix III $65 \mathrm{~nm}$ FPGA. In most cases, we implemented the equivalent FPGA circuits for comparison. Power consumption is not compared due to the scarcity of data in the literature and the difficulty in standardizing testing conditions such as test vectors, voltage, and temperature.

We normalize area measurements to a $65 \mathrm{~nm}$ process using an ideal scale factor of $0.5 \times$ area between process nodes. We normalize delay using published ring oscillator data, with

\begin{tabular}{cccc}
\hline & $90 \mathrm{~nm}$ & $65 \mathrm{~nm}$ & $45 \mathrm{~nm}$ \\
\hline Area & 0.5 & 1.0 & 2.0 \\
Delay & 0.78 & 1.0 & 1.23 \\
\hline
\end{tabular}

Table 1: Normalization Factors Between Processes

\begin{tabular}{lcc}
\hline Resource & $\begin{array}{c}\text { Relative Area } \\
\text { (Equiv. LABs) }\end{array}$ & $\begin{array}{c}\text { Tile Area } \\
\left(\mathrm{mm}^{2}\right)\end{array}$ \\
\hline LAB & 1 & 0.0221 \\
ALUT (half-ALM) & 0.05 & 0.0011 \\
M9K memory & 2.87 & 0.0635 \\
M144K memory & 26.7 & 0.5897 \\
DSP block & 11.9 & 0.2623 \\
\hline Total core area & 18621 & 412 \\
\hline
\end{tabular}

Table 2: Estimated FPGA Resource Area Usage

the understanding that these reflect gate delay scaling more than interconnect scaling. Intel reports $29 \%$ FO1 delay improvement between $90 \mathrm{~nm}$ and $65 \mathrm{~nm}$, and 23\% FO2 delay improvement between $65 \mathrm{~nm}$ and $45 \mathrm{~nm}[12,13]$. The area and delay scaling factors used are summarized in Table 1.

Delay is measured as the longest register to register path or input to output path in a circuit. In papers that describe CMOS circuits embedded in a larger unit (e.g., a shifter inside an ALU), we conservatively assume that the subcircuit has the same cycle time as the larger unit. In FPGA circuits, delay is measured using register to register paths, with the register delay subtracted out when comparing circuits that do not include a register (e.g., wire delay).

To measure FPGA resource usage, we implement circuits on the Stratix III FPGA and use the "logic utilization" metric as reported by Quartus II to account for lookup tables (LUT) and registers, and count partially-used memory and multiplier blocks as entirely used since it is unlikely another part of the design can use them. Table 2 shows the areas of the Stratix III FPGA resources. The FPGA tile areas include routing area so we do not track routing separately. The core of the the largest Stratix III (EP3LS340) FPGA contains 13500 LABs of 10 ALMs each, 1040 M9K memories, $48 \mathrm{M} 144 \mathrm{~K}$ memories, and 72 DSP blocks, for a total of $18621 \mathrm{LAB}$ equivalent areas and $412 \mathrm{~mm}^{2}$ core area.

We implemented FPGA circuits using Altera Quartus II 10.0 SP1 using the fastest speed grade of the largest Stratix III. Circuits containing MLABs used Stratix IV due to hardware $^{1}$ issues. Registers delimit the inputs and outputs of our circuits under test. We set timing constraints to maximize clock speed, reflecting the use of these circuits as part of a larger circuit in the FPGA core.

\subsection{Additional Limitations}

The trade-off space for a given circuit structure on custom CMOS is huge and our comparison circuit examples may not all have the same optimization targets. Delay, area, power, and design effort can all be traded-off, resulting in vastly different circuit performance. We assume that designs published in the literature are optimized primarily for delay with reasonable values for the other metrics and we implement our FPGA equivalents with the same approach.

Another source of imprecision results from the differences in performance between chip manufacturers. For example,

\footnotetext{
${ }^{1}$ A Stratix III erratum halves the MLAB capacity to 320 bits and is fixed in Stratix IV. See Stratix III Device Family Errata Sheet
} 
transistor drive strength impacts logic gate delay and is voltage- and process-dependent. At $100 \mathrm{nA} / \mu \mathrm{m}$ leakage current, Intel's $65 \mathrm{~nm}$ process [14] achieves 1.46 and $0.88 \mathrm{~mA} / \mu \mathrm{m}$ at $1.2 \mathrm{~V}$ for NMOS and PMOS, respectively, and 1.1 and $0.66 \mathrm{~mA} / \mu \mathrm{m}$ at $1.0 \mathrm{~V}$, while TSMC's $65 \mathrm{~nm}$ process [15] achieves 1.01 and $0.48 \mathrm{~mA} / \mu \mathrm{m}$ at $1.0 \mathrm{~V}$. The choice of supply voltage results from both the power-performance tradeoff and the transistor reliability the manufacturer can achieve.

Finally, we note that high-performance microprocessor designs tend to have generous power budgets that FPGAs do not enjoy, and this has an impact that is embedded in the area and speed comparisons.

\section{FPGA VS. CUSTOM CMOS}

\subsection{Complete Processor Cores}

We begin by comparing complete processor cores implemented on an FPGA vs. custom CMOS to provide context for the building block measurements. Table 3 shows a comparison of the area and delay of four commercial processors that have both custom CMOS and FPGA implementations and includes in-order, multithreaded, and complex out-oforder processors. The FPGA implementations are synthesized from RTL code for the custom CMOS processors, with some FPGA-specific circuit-level optimizations. However, the FPGA-specific optimization effort is smaller than for custom CMOS designs and could inflate the area and delay ratios slightly. Overall, custom processors have delay ratios of $18-26 \times$ and area ratios of $17-27 \times$, with no obvious trends with processor complexity.

The OpenSPARC T1 and T2 cores are derived from the Sun UltraSPARC T1 and T2, respectively [21]. Both cores are in-order multithreaded, and use the 64-bit SPARC V9 instruction set. The OpenSPARC T2 has the encryption unit from the UltraSPARC T2 removed, although we believe the change is small enough to not significantly skew our measurements.

The Intel Atom is a reduced-voltage dual-issue in-order 64-bit x86 processor with two-way multithreading. The FPGA synthesis by Wang et al. includes only the processor core without L2 cache, and occupies $85 \%$ of the largest $65 \mathrm{~nm}$ Virtex-5 FPGA (XC5VLX330) [10]. Our FPGA area metric assumes the core area of the largest Virtex 5 is the same as the Stratix III, $412 \mathrm{~mm}^{2}$.

The Intel Nehalem is a high-performance multiple-issue out-of-order 64 -bit x86 processor with two-way multithreading. The Nehalem's core area was estimated from a die photo. The FPGA synthesis by Schelle et al. does not include the per-core L2 cache, and occupies roughly $300 \%$ of the largest Virtex-5 FPGA [19]. Because the FPGA synthesis is split over multiple chips and runs at $520 \mathrm{kHz}$, it is not useful for estimating delay ratio.

\subsection{SRAM Blocks}

SRAM blocks are commonly used in processors for building caches and register files. SRAM performance can be characterized by latency and throughput. Custom SRAM designs can trade latency and throughput by pipelining, while FPGA designs are typically limited to the types of hard SRAM blocks that exist on the FPGA. SRAMs on the Stratix III FPGA can be implemented using two sizes of hard block SRAM, LUTRAM, or in registers and LUTs. Their throughput and density are compared in Table 4 to five high-performance custom SRAMs in $65 \mathrm{~nm}$ processes.

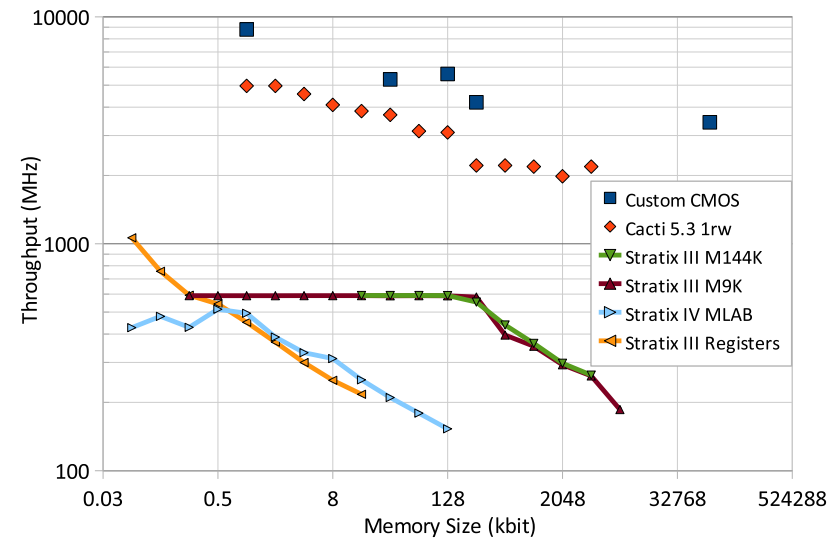

Figure 1: SRAM Throughput

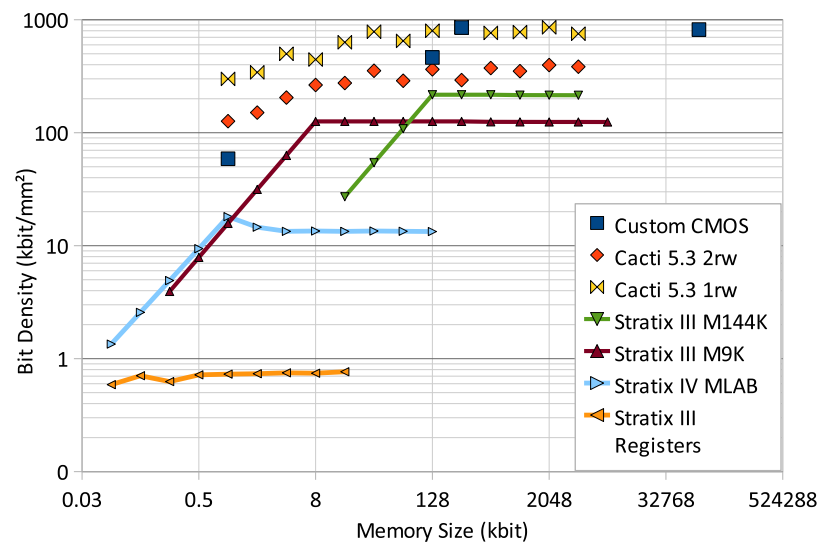

Figure 2: SRAM Density

The density and throughput of FPGA and custom SRAMs is plotted against memory size in Figures 1 and 2. The plots include data from CACTI 5.3, a CMOS memory performance and area model [27]. The throughput ratio between FPGA memories and custom is $7-10 \times$, lower than the overall delay ratio of $18-26 \times$, showing that SRAMs are relatively fast on FPGAs. It is surprising that this ratio is not lower because FPGA SRAM blocks have little programmability. The FPGA data above use 32-bit wide data ports that slightly underutilize the native 36 -bit ports. The raw density of an FPGA SRAM block is listed in Table 4.

Below 9 kbit, the bit density of FPGA RAMs falls off nearly linearly with reducing RAM size because M9Ks are underutilized, and the MLAB density is low. For larger arrays with good utilization, FPGA SRAM arrays have a density ratio of only $2-5 \times$ vs. single read-write port (1rw) CMOS (and CACTI) SRAMs, far below the full processor area ratio of $17-27 \times$.

As FPGA SRAMs use dual-ported (2rw) arrays, we also plotted CACTI's 2rw model for comparison. For arrays of similar size, the bit density of CACTI's 2rw models are $1.9 \times$ and $1.5 \times$ the raw bit density of fully-utilized M9K and M144K memory blocks, respectively. This suggests that half of the bit density gap between FPGA and custom in our single-ported test is due to FPGA memories paying the overhead of dual ports.

For register file use where latency may be more important than memory density, custom processors have the option of trading throughput for area and power by using faster and larger storage cells. The $65 \mathrm{~nm}$ Pentium 4 register file trades $5 \times$ bit density for $9 \mathrm{GHz}$ single-cycle performance 


\begin{tabular}{|c|c|c|c|c|c|c|c|}
\hline \multirow[b]{2}{*}{ Processor } & \multicolumn{2}{|c|}{ Custom CMOS } & \multicolumn{2}{|c|}{ FPGA } & \multirow{2}{*}{$\begin{array}{c}\text { Logic } \\
\text { Utilization } \\
(\mathrm{ALUT}) \\
\end{array}$} & \multicolumn{2}{|c|}{ Ratios } \\
\hline & $\begin{array}{r}\mathrm{f}_{\max } \\
(\mathrm{MHz})\end{array}$ & $\begin{array}{r}\text { Area } \\
\left(\mathrm{mm}^{2}\right)\end{array}$ & $\begin{array}{r}\mathrm{f}_{\max } \\
(\mathrm{MHz})\end{array}$ & $\begin{array}{r}\text { Area } \\
\left(\mathrm{mm}^{2}\right)\end{array}$ & & $\mathrm{f}_{\max }$ & Area \\
\hline OpenSPARC T1 $(90 \mathrm{~nm})$ [16] & 1800 & 6.0 & 79 & 100 & 86597 & 23 & 17 \\
\hline OpenSPARC T2 $(65 \mathrm{~nm})[17]$ & 1600 & 11.7 & 88 & 294 & 250235 & 18 & 25 \\
\hline Atom $(45 \mathrm{~nm})[10,18]$ & $>1300$ & 12.8 & 50 & 350 & $85 \%$ & 26 & 27 \\
\hline Nehalem $(45 \mathrm{~nm})[19,20]$ & 3000 & 51 & - & 1240 & $300 \%$ & - & 24 \\
\hline Geometric Mean & & & & & & 22 & 23 \\
\hline
\end{tabular}

Table 3: Complete Processor Cores. Area and delay normalized to $65 \mathrm{~nm}$.

\begin{tabular}{lcrccccc}
\hline Design & Ports & $\begin{array}{c}\text { Size } \\
(\mathrm{kbit})\end{array}$ & $\begin{array}{c}\mathrm{f}_{\max } \\
(\mathrm{MHz})\end{array}$ & $\begin{array}{c}\text { Area } \\
\left(\mathrm{mm}^{2}\right)\end{array}$ & $\begin{array}{c}\text { Bit Density } \\
\left(\mathrm{kbit} / \mathrm{mm}^{2}\right)\end{array}$ & \multicolumn{2}{c}{ Ratios } \\
$\mathrm{f}_{\max }$ & Density \\
\hline IBM 6T 65 nm [22] & 2r or 1w & 128 & $56001.2 \mathrm{~V}$ & 0.276 & 464 & 9.5 & 2.1 \\
Intel 6T 65 nm [23] & 1rw & 256 & $42001.2 \mathrm{~V}$ & 0.3 & 853 & 7.6 & 3.9 \\
Intel 6T 65 nm [14] & 1rw & $70 \mathrm{Mb}$ & $34301.2 \mathrm{~V}$ & $110[24]$ & 820 & - & - \\
IBM 8T 65 nm SOI [25] & 1r1w & 32 & $53001.2 \mathrm{~V}$ & - & - & 9.0 & - \\
Intel 65 nm Regfile [26] & 1r1w & 1 & $88001.2 \mathrm{~V}$ & 0.017 & 59 & 18 & 3.3 \\
\hline Registers & 1r1w & - & - & - & 0.77 & & \\
MLAB & 1r1w & $640 \mathrm{~b}$ & $\sim 600$ & 0.033 & 23 & & \\
M9K & 1r1w & 9 & 590 & 0.064 & 142 & \\
M144K & 1r1w & 144 & 590 & 0.59 & 244 & & \\
\hline
\end{tabular}

Table 4: Custom and FPGA SRAM Blocks

\begin{tabular}{lcccccc}
\hline & \multicolumn{2}{c}{ CACTI 5.3 } & \multicolumn{2}{c}{ FPGA } & \multicolumn{2}{c}{ Ratios } \\
Ports & $\begin{array}{c}\mathrm{f}_{\mathrm{max}} \\
(\mathrm{MHz})\end{array}$ & $\begin{array}{c}\text { Density } \\
\left(\frac{\mathrm{kbit}^{2}}{\mathrm{~mm}^{2}}\right)\end{array}$ & $\begin{array}{c}\mathrm{f}_{\mathrm{max}} \\
(\mathrm{MHz})\end{array}$ & $\begin{array}{c}\text { Density } \\
\left(\frac{\mathrm{kbit}}{\mathrm{mm}^{2}}\right)\end{array}$ & $\mathrm{f}_{\max }$ & Density \\
\hline 2r1w & 4430 & 109 & 501 & 15.7 & 9 & 7 \\
4r2w & 4200 & 35 & 320 & 0.25 & 13 & 143 \\
6r3w & 3800 & 17 & 286 & 0.20 & 13 & 89 \\
8r4w & 3970 & 9.8 & 269 & 0.15 & 15 & 65 \\
10r5w & 3740 & 6.3 & 266 & 0.10 & 14 & 61 \\
12r6w & 3520 & 4.5 & 249 & 0.090 & 14 & 51 \\
14r7w & 3330 & 3.4 & 237 & 0.080 & 14 & 43 \\
16r8w & 3160 & 2.7 & 224 & 0.068 & 14 & 39 \\
\hline Live Value Table (LVT) & & & & & \\
4r2w & & & 420 & 1.50 & 10 & 23 \\
8r4w & & & 345 & 0.41 & 12 & 24 \\
\hline
\end{tabular}

Table 5: Multiported 2 kbit SRAM. LVT data from LaForest et al. [28].

[26]. FPGA RAMs lack this flexibility, and the delay ratio is even greater $(18 \times)$ for this specific use.

\subsection{Multiported SRAM Blocks}

FPGA hard SRAM blocks can typically implement up to two read-write ports (2rw). Increasing the number of read ports can be achieved reasonably efficiently by replicating the memory blocks but increasing the number of write ports cannot. A multi-write port RAM can be implemented using registers for storage, but it is inefficient. A more efficient method using hard RAM blocks for most of the storage replicates memory blocks for each write and read port and uses a live value table (LVT) to indicate for each word which of the replicated memories holds the most recent copy [28]. We present data for multiported RAMs implemented using registers, LVT-based multiported memories [28], and CACTI 5.3 models of custom CMOS multiported RAMs. We focus on a $64 \times 32$-bit ( 2 kbit) memory array with twice as many read ports as write ports ( $2 N$ read, $N$ write) because it is similar to register files in processors. Table 5 shows the throughput and density comparisons.

The custom vs. FPGA bit density ratio is $7 \times$ for $2 \mathrm{r} 1 \mathrm{w}$, and increases to $23 \times$ and $143 \times$ for $4 \mathrm{r} 2 \mathrm{w}$ LVT- and registerbased memories, respectively. The delay ratio is $9 \times$ for $2 \mathrm{r} 1 \mathrm{w}$, and increases to $10 \times$ and $13 \times$ for $4 \mathrm{r} 2 \mathrm{w}$ LVT- and registerbased memories, respectively, a smaller impact than the area ratio increase.

\subsection{Content-Addressable Memories}

A Content-Addressable Memory (CAM) is a logic circuit that allows associative searches of its stored contents. Custom CMOS CAMs are implemented as dense arrays using $9 \mathrm{~T}$ to $11 \mathrm{~T}$ cells compared to $6 \mathrm{~T}$ in SRAM, and are about $2-3 \times$ less dense than SRAMs. Ternary CAMs use two storage cells per "bit" to store three states $(0,1$, and don't-care), a capability often used for longest prefix searches in network packet routers. In processors, CAMs are used in tag arrays for highassociativity caches and TLBs. CAM-like structures are also used in out-of-order instruction schedulers. CAMs in processors require both frequent read and write capability, but not large capacities. Pagiamtzis and Sheikholeslami give a good overview of the CAM design space [29].

There are several methods of implementing CAM functionality on FPGAs [30]. CAMs implemented in soft logic use registers for storage and LUTs to read, write, and search the stored bits. Another proposal, which we call BRAMCAM, stores one-hot encoded match-line values in block RAM to provide the functionality of a $w \times b$-bit CAM using a $2^{b} \times w$-bit block RAM [31]. The soft logic CAM is the only design that provides one-cycle writes, while the BRAMCAM offers improved bit density with two-cycle writes. We do not consider CAM implementations with even longer write times.

Table 6 shows a variety of custom CMOS and FPGA CAM designs. Figures 3 and 4 plot these and also 8-bit 


\begin{tabular}{|c|c|c|c|c|c|}
\hline \multirow[b]{2}{*}{ Ternary CAMs } & $\begin{array}{r}\text { Size } \\
\text { (bits) }\end{array}$ & $\begin{array}{l}\text { Searcl } \\
\text { Time } \\
(\mathrm{ns})\end{array}$ & $\begin{array}{l}\text { Bit } \\
\text { Density } \\
\left(\frac{\mathrm{kbit}}{\mathrm{mm}^{2}}\right)\end{array}$ & \multicolumn{2}{|c|}{ Ratios } \\
\hline & & & & & \\
\hline IBM $64 \times 72[32]$ & 4608 & 0.6 & - & 5.0 & - \\
\hline IBM $64 \times 240[32]$ & 15360 & 2.2 & 167 & 1.7 & 205 \\
\hline \multicolumn{6}{|l|}{ Binary CAMs } \\
\hline POWER6 8×60 [33] & 480 & $<0.2$ & - & 14 & - \\
\hline Godson-3 64×64[34] & 4096 & 0.55 & 76 & 5 & 99 \\
\hline Intel $64 \times 128[35]^{2}$ & 8192 & 0.25 & 167 & 14 & 209 \\
\hline \multicolumn{6}{|c|}{ FPGA Binary CAMs } \\
\hline Soft logic $64 \times 72$ & 4608 & 3.0 & 0.82 & & \\
\hline Soft logic $64 \times 240$ & 15360 & 3.8 & 0.81 & & \\
\hline Soft logic $8 \times 60$ & 480 & 2.1 & 0.83 & & \\
\hline Soft logic $64 \times 64$ & 4096 & 2.9 & 0.77 & & \\
\hline Soft logic $64 \times 128$ & 8192 & 3.4 & 0.80 & & \\
\hline MLAB-CAM $64 \times 20$ & 1280 & 4.5 & 1.0 & & \\
\hline M9K-CAM $64 \times 16$ & 1024 & 2.0 & 2.0 & & \\
\hline
\end{tabular}

Table 6: CAM Designs

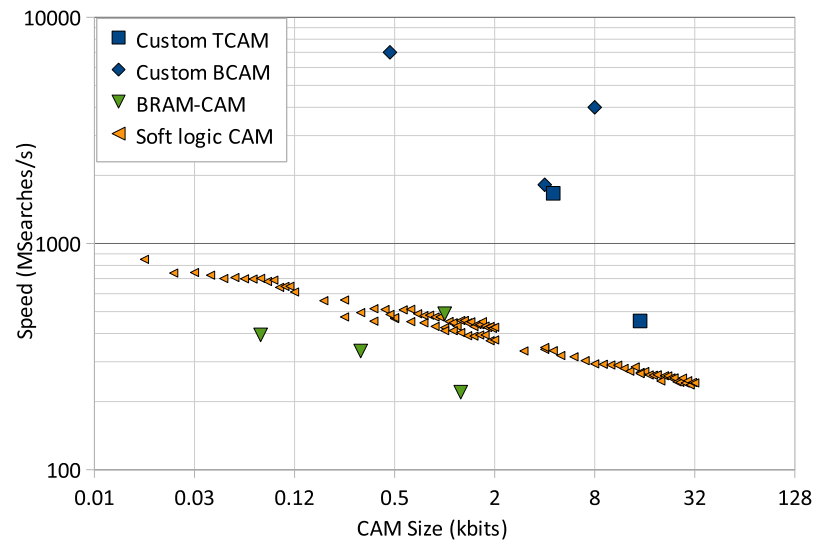

Figure 3: CAM Search Speed

and 128-bit soft logic CAMs of varying depth. Because of high power consumption, CAMs often have three competing design goals: delay, density, and energy per bit per search. CAMs can achieve delay comparable to SRAMs but at a high cost in power. Both the POWER6 TLB and Intel's $64 \times 128$ BCAM achieve at least $4 \mathrm{GHz}$, but the latter uses $13 \mathrm{fJ} / \mathrm{bit} / \mathrm{search}$ while IBM's $450 \mathrm{MHz} 64 \times 240$ TCAM uses $1 \mathrm{fJ} / \mathrm{bit} / \mathrm{search}$. While Intel's BCAM and the Godson-3 TLB are both 64-entry full-custom designs, Intel achieves twice the bit density and half the cycle time at equal energy per bit per search, highlighting the impact of good circuit design, layout, and process technology.

As shown in Table 6, soft logic CAMs have poor 100$210 \times$ bit density ratios vs. custom CAMs. Despite the low density, the delay ratio is only $14 \times$. BRAM-CAMs built from M9Ks can offer $2.4 \times$ better density than soft logic CAMs but halved write bandwidth. Despite the higher port-width/depth aspect ratio, MLAB BRAM-CAMs have bit density worse than M9K-CAMs because of control logic overhead. The halved write bandwidth of BRAM-CAMs make them unsuitable for performance-critical uses, such as tag matching in instruction schedulers and L1 caches.

We observe that the bit density of soft logic CAMs is nearly the same as using registers to implement RAM (Table 4), suggesting that the area inefficiency comes from using registers for storage, not the associative search itself.

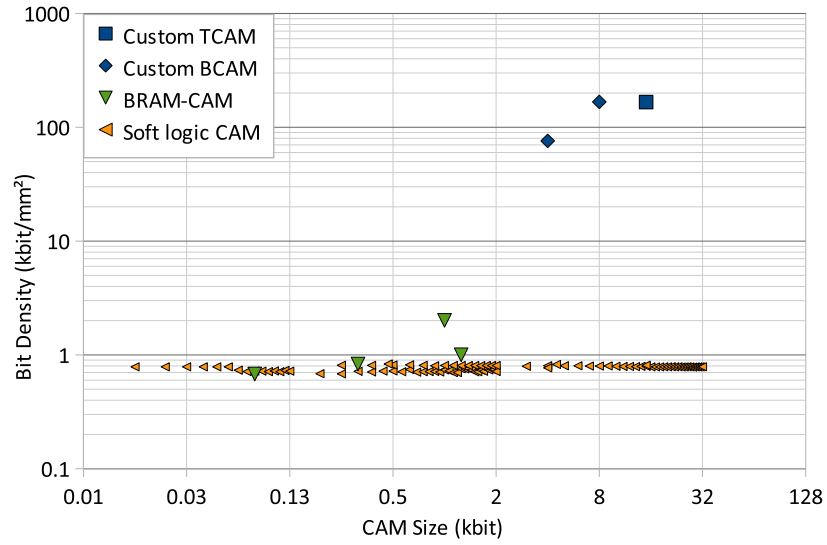

Figure 4: CAM Bit Density

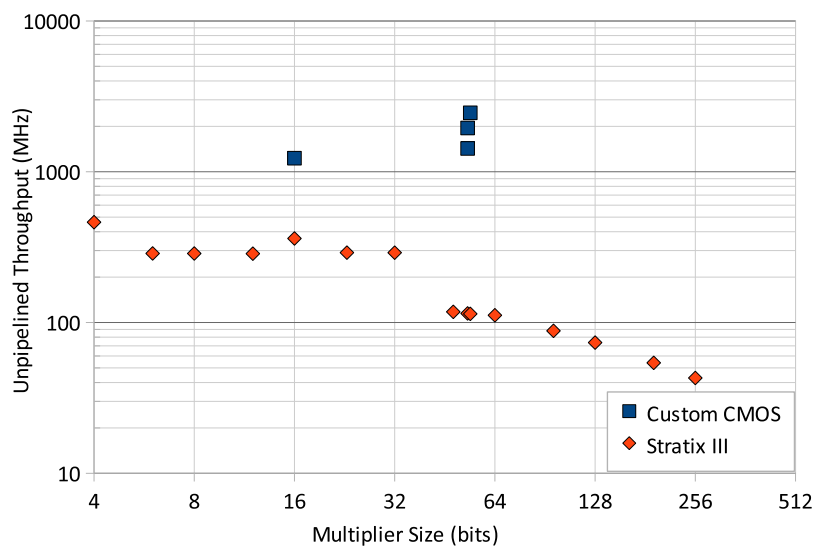

Figure 5: Multiplier Latency

\subsection{Multipliers}

Multiplication is an operation performed frequently in signal processing applications, but not used as often in processors. Multiplier blocks can also be used to inefficiently implement shifters and multiplexers [39].

Figure 5 shows the latency of multiplier circuits on custom CMOS and on FPGA using hard DSP blocks. Latency is the product of the cycle time and the number of pipeline stages, and does not adjust for unbalanced pipeline stages or pipeline latch overheads. Table 7 shows details of the design examples. The two IBM multipliers have latency ratios comparable to full processor cores. Intel's 16-bit multiplier design has much lower latency ratios as it appears to target low power instead of delay. Because custom multiplier de-

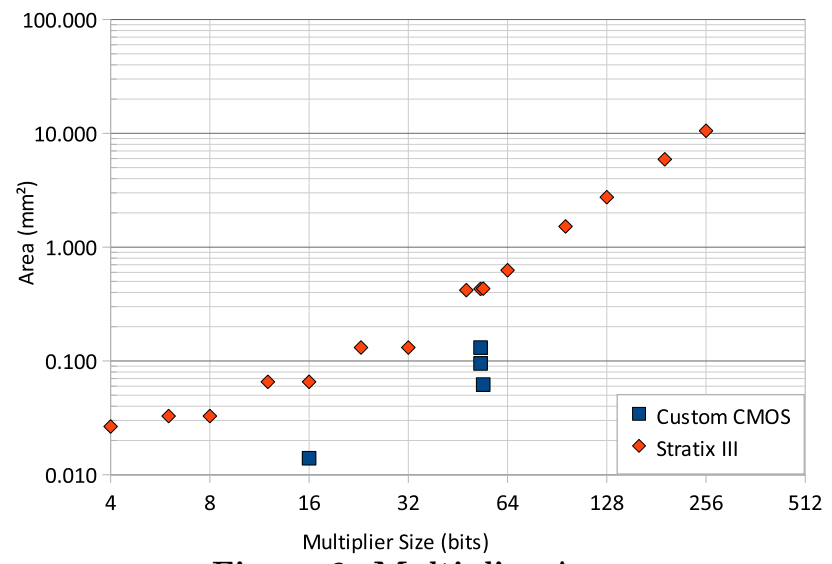

Figure 6: Multiplier Area 


\begin{tabular}{|c|c|c|c|c|c|c|}
\hline Design & Size & Stages & $\begin{array}{l}\text { Latency } \\
\text { S (ns) }\end{array}$ & $\begin{array}{c}\text { Area } \\
\left(\mathrm{mm}^{2}\right)\end{array}$ & $\begin{array}{r}\mathrm{Ra} \\
\text { Laten }\end{array}$ & $\begin{array}{l}\text { As } \\
\text { Area }\end{array}$ \\
\hline $\begin{array}{l}\text { Intel } 90 \mathrm{~nm} \\
1.3 \mathrm{~V}[36]\end{array}$ & $16 \times 16$ & 1 & 0.81 & 0.014 & 3.4 & 4.7 \\
\hline $\begin{array}{l}\text { IBM } 90 \mathrm{~nm} \\
\text { SOI } 1.4 \mathrm{~V}[37]\end{array}$ & $54 \times 54$ & 4 & 0.41 & 0.062 & 22 & 7.0 \\
\hline $\begin{array}{l}\text { IBM } 90 \mathrm{~nm} \\
\text { SOI } 1.3 \mathrm{~V}[38]\end{array}$ & $53 \times 53$ & 3 & 0.51 & 0.095 & 17 & 4.5 \\
\hline Stratix III & $16 \times 16$ & 1 & 2.8 & 0.066 & & \\
\hline Stratix III & $54 \times 54$ & 1 & 8.8 & 0.43 & & \\
\hline
\end{tabular}

Table 7: Multiplier area and delay, normalized to $65 \mathrm{~nm}$ process. Unpipelined latency is pipelined cycle time $\times$ stages.

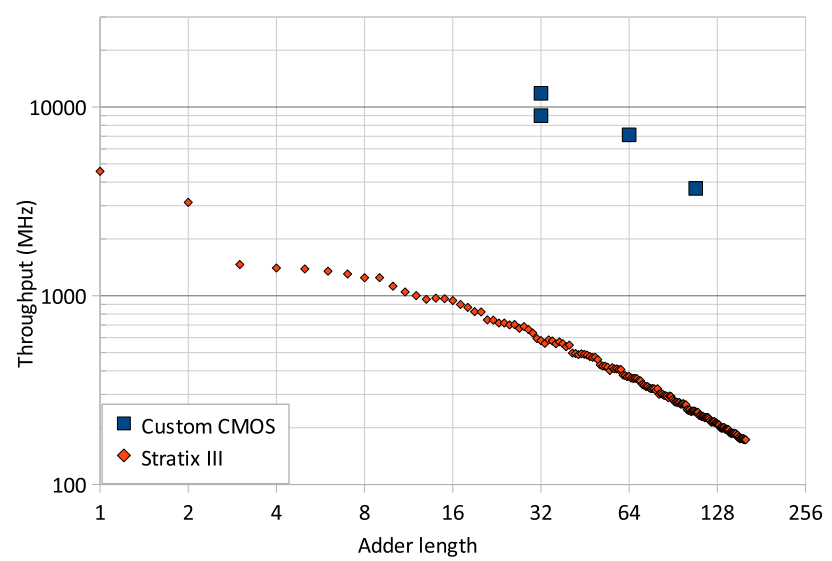

Figure 7: Adder Delay

signs are often more deeply pipelined (3 and 4 stages) than the hard multipliers on FPGAs (2 stages), throughput ratios are higher than latency ratios.

The area of the custom CMOS and FPGA multipliers are plotted in Figure 6. The area ratios of $4.5-7.0 \times$ are much lower than for full processor cores.

\subsection{Adders}

Custom CMOS adder circuit designs can span the areadelay trade-off space from slow ripple-carry adders to fast parallel adders. On an FPGA, adders are usually implemented using hard carry chains that implement variations of the ripple-carry adder, although carry-select adders have been also been used. Although parallel adders can be implemented with soft logic and routing, the lack of dedicated circuitry means parallel adders are bigger and slower than the ripple-carry adder with hard carry chains [44].

Figure 7 plots a comparison of adder delay, with details in

\begin{tabular}{lccccc}
\hline Design & $\begin{array}{c}\text { Size } \\
(\mathrm{bit})\end{array}$ & $\begin{array}{c}\mathrm{f}_{\max } \\
(\mathrm{MHz})\end{array}$ & $\begin{array}{c}\text { Area } \\
\left(\mathrm{mm}^{2}\right)\end{array}$ & $\begin{array}{c}\text { Delay } \\
\text { Ratio }\end{array}$ & $\begin{array}{c}\text { Area } \\
\text { Ratio }\end{array}$ \\
\hline Agah et al. [40] & 32 & $120001.3 \mathrm{~V}$ & - & 20 & - \\
Kao et al. & 64 & $71001.3 \mathrm{~V}$ & 0.016 & 19 & 4.5 \\
90 nm [41] & 32 & $90001.3 \mathrm{~V}$ & - & 16 & - \\
Pentium 4 [42] & 108 & $37001.0 \mathrm{~V}$ & 0.017 & 15 & 6.9 \\
IBM [43] & 32 & 593 & 0.035 & & \\
\hline & 64 & 374 & 0.071 & & \\
Stratix III & 108 & 242 & 0.119 & & \\
&
\end{tabular}

Table 8: Adders. Area and delay normalized to $65 \mathrm{~nm}$ process.

\begin{tabular}{rcccc}
\hline \multirow{2}{*}{$\begin{array}{c}\text { Mux } \\
\text { Inputs }\end{array}$} & \multicolumn{2}{c}{ Area } & Delay & \multicolumn{2}{c}{ Custom } \\
& $\left(\mathrm{mm}^{2}\right)$ & $(\mathrm{ps})$ & $(\mathrm{ps})$ & $\begin{array}{c}\text { Delay } \\
\text { Ratio }\end{array}$ \\
\hline 2 & 0.0011 & 210 & 2.8 & 74 \\
4 & 0.0011 & 260 & 4.9 & 53 \\
8 & 0.0022 & 500 & 9.1 & 54 \\
16 & 0.0055 & 680 & 18 & 37 \\
32 & 0.0100 & 940 & 29 & 32 \\
64 & 0.0232 & 1200 & 54 & 21 \\
\hline
\end{tabular}

Table 9: Analytical model of passive tree multiplexers [45] normalized to $65 \mathrm{~nm}$ process.

Table 8 . The Pentium 4 delay is conservative as the delay is for the full integer ALU. FPGA adders achieve delay ratios of $15-20 \times$ and a low area ratio of around $4.5-7 \times$.

\subsection{Multiplexers}

Multiplexers are found in many circuits, yet we have found little literature that provides their area and delay in custom CMOS. Instead, we estimate delays of small multiplexers using an RC analytical model and the delays of the Pentium 4 shifter unit and the Stratix III ALM. Our area ratio estimate comes from an indirect measurement using the ALM.

Table 9 shows a delay comparison between FPGA and an analytical model of switch-based tree multiplexers [45]. This passive switch model is pessimistic for larger multiplexers, as active buffer elements can reduce delay. On an FPGA, small multiplexers can often be combined with other logic with minimal extra delay and area, so multiplexers measured in isolation are likely pessimistic. For small multiplexers, the delay ratio is high, roughly $40-75 \times$. Larger multiplexers appear to have decreasing delay ratios, but we believe this is largely due to the unsuitability of passive designs.

The $65 \mathrm{~nm}$ Pentium 4 integer shifter datapath [42] is dominated by small multiplexers (sizes 3,4 , and 8 ), but not in isolation. We implemented the datapath (Figure 8) excluding control logic on the Stratix III, with results shown in Table 10. The delay ratio of $20 \times$ is smaller than suggested by the isolated multiplexer comparison, but may be optimistic if Intel omitted details from their shifter circuit diagram. Another delay ratio estimate can be made by examining the Stratix III Adaptive Logic Module itself, as its delay consists mainly of multiplexers. Configuration RAM hold static values and do not affect delay. We implemented a circuit equivalent to an ALM as described in the Stratix III Handbook [46], comparing delays of the FPGA implementation to custom CMOS delays of the ALM given by the Quartus timing models. Internal LUTs are modeled as multiplexers. Each ALM input pin is modeled as a 21-to-1 multiplexer, as 21 to 30 are reasonable sizes according to Lewis et al. [47].

We examined one long path and one short path out of many possible timing paths through the ALM. The long and short timing paths begin after the input multiplexers for pins datab0 and dataf0, respectively, both terminating at the LUT register. We placed these two paths into separate clock domains to ensure they were independently optimized. Table 10 shows delay ratios of $7.1 \times$ and $11.7 \times$ for the long and short paths, respectively. These delay ratios are lower compared to previous examples due to the lower power and area budgets preventing custom FPGAs from being as aggressively delay-optimized as custom processors, and to 


\begin{tabular}{lccc}
\hline Circuit & $\begin{array}{c}\text { FPGA } \\
\text { Delay (ps) }\end{array}$ & $\begin{array}{c}\text { Custom } \\
\text { Delay } \\
(\mathrm{ps})\end{array}$ & Ratio \\
\hline 65 nm Pentium 4 Shifter & 2260 & 111 & 20 \\
\hline Stratix III ALM & & & \\
Long path & 2500 & 350 & 7.1 \\
Short path & 800 & 68 & 11.7 \\
\hline
\end{tabular}

Table 10: Delay of Multiplexer-Dominated Circuits

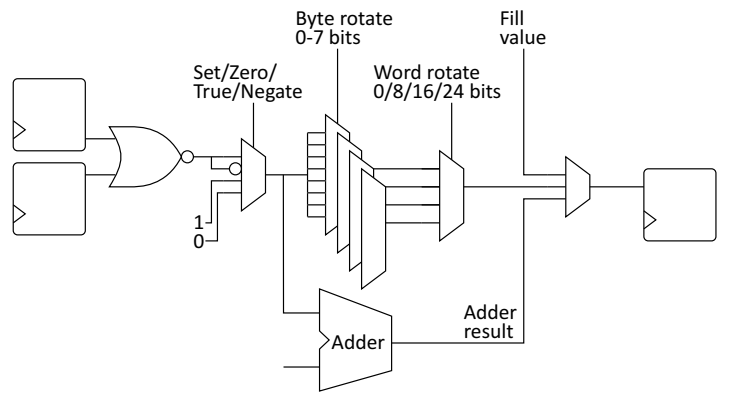

Figure 8: 65 nm Pentium 4 Integer ALU Shifter [42]

extra circuit complexity not shown in the Stratix III Handbook.

We estimate the multiplexer area ratio indirectly by again implementing the equivalent circuit of an ALM on a Stratix III. We find that our equivalent ALM consumes 104 ALUTs, or roughly 52 ALMs. An area ratio of $52 \times$ is the ratio of the area of the FPGA equivalent circuit (52 ALMs) compared to the silicon area of the custom circuit (1 ALM equivalent). The real area ratio is substantially greater, as we implemented only the ALM's input and internal multiplexers and did not include global routing resources or configuration RAM. A rule of thumb is that half of an FPGA's core area is spent in the programmable global routing network, for an area ratio estimate of $104 \times$. We expect this to be even higher once configuration RAM and other ALM complexities are accounted for.

Groups of multiplexers have delay ratios below $20 \times$, with small isolated multiplexers being worse $(40-75 \times)$. However, multiplexers are particularly area-intensive with an area ratio greater than $100 \times$. Thus we find that the intuition that multiplexers are expensive is justified, especially from an area perspective.

\subsection{Pipeline Latches}

In synchronous circuits, the maximum clock speed of a circuit is typically limited by a register-to-register delay path from a pipeline latch ${ }^{2}$, through a pipeline stage's combinational logic, to the next set of pipeline latches. The delay of a pipeline latch (setup and clock-to-output times) impacts the speed of a circuit and the clock speed improvement when increasing pipeline depth.

The "effective" cost of inserting an extra pipeline register into LUT-based pipeline logic is measured by observing the increase in delay as the number of LUTs between registers increases, then extrapolating the delay to zero LUTs. Table 11 shows estimates of the delay cost of a custom CMOS pipeline stage. The $180 \mathrm{~nm}$ Pentium 4 design assumed $90 \mathrm{ps}$ of pipeline latch delays including clock skew [48], which we

\footnotetext{
${ }^{2}$ Latch refers to pipeline storage elements. This can be a latch, flip-flop, or other implementation.
}

\begin{tabular}{lcc}
\hline Design & Register Delay (ps) & Delay Ratio \\
\hline Sprangle et al. [48] & 35 (90 ps in 180 nm) & 12 \\
Hartstein et al. [49] & $32(2.5$ FO4) & 14 \\
Hrishikesh et al. [50] & $23(1.8$ FO4) & 19 \\
\hline Geometric Mean & 29.5 & 15 \\
\hline Stratix III & 436 & - \\
\hline
\end{tabular}

Table 11: Pipeline Latch Delay

scaled according to the FO1 ring oscillator delays for Intel's processes (11 ps at $180 \mathrm{~nm}$ to $4.25 \mathrm{ps}$ at $65 \mathrm{~nm}$ ) [14]. Hartstein et al. and Hrishikesh et al. present estimates expressed in FO4 delays, which were scaled to an estimated FO4 delay of $12.8 \mathrm{ps}$ for Intel's $65 \mathrm{~nm}$ process.

The delay ratio for a pipeline latch is $10-15 \times$. Although we do not have area comparisons, registers are considered to occupy very little FPGA area because more LUTs are used than registers in most FPGA circuits, yet FPGA logic elements include at least one register for every LUT.

\subsection{Point-to-Point Routing}

Interconnect delay comprises a significant portion of the total delay in both FPGAs and modern CMOS processes. Figure 9 plots the point-to-point wire delays for three classes of interconnect (local, intermediate, and global), and FPGA routing delay both in absolute distance and normalized for decreased FPGA area efficiency.

We model each buffered segment as an RC delay as illustrated in Figure 10 and Equation 1, where $h$ is the buffer transistor size and $R_{t r}$ is the linearized buffer resistance. Buffered wires are modeled as multiple identical segments with the number of segments chosen to minimize delay, while minimum-delay buffer transistor sizing can be found by differentiating Equation 1 with respect to $h$. Equation 1 is modified from Bakoglu and Meindl to include transistor gate $\left(C_{g}\right)$ and junction $\left(C_{d}\right)$ lumped capacitances and to model $50 \%$-to-50\% delay instead of 0-to-90\% [51].

$$
T=R_{t r} C_{d}+\left(\frac{1}{h} R_{t r}+\frac{1}{2} R_{i n t}\right) C_{i n t}+\left(\frac{1}{h} R_{t r}+R_{i n t}\right) h C_{g}
$$

We use interconnect and transistor parameters from the International Technology Roadmap for Semiconductors (ITRS) 2007 report [52]. We model the minimum pitch for each class of wiring (local, intermediate, global). Buffers are assumed to be inverters with 2:1 PMOS to NMOS transistor size ratio, and junction capacitance is approximated as half of gate capacitance. Gate and junction capacitances are modeled as lump capacitances and wires are modeled as distributed RC. Interconnect delays using ITRS 2007 data may be pessimistic, as Intel's $65 \mathrm{~nm}$ process reports lower delays using larger pitch and wire thicknesses [14].

The point-to-point delay on FPGA is measured using the delay between two manually-placed registers, with the delay of the register itself subtracted out, and includes the overhead of routing programmability. Assuming LABs have an aspect ratio (the vertical/horizontal ratio of delay for each LAB) of 1.6 gives a good delay vs. Manhattan distance fit. The physical distance is calculated from LAB coordinates using the aspect ratio and area of a LAB (Table 2).

In Figure 9, the delay of the CMOS load and driving buffers (one FO1) dominates the delay for CMOS short wire lengths under $20 \mu \mathrm{m}$. FPGA short local wires $(100 \mu \mathrm{m})$ have a delay ratio around $9 \times$. Long global wire delay is quite close 


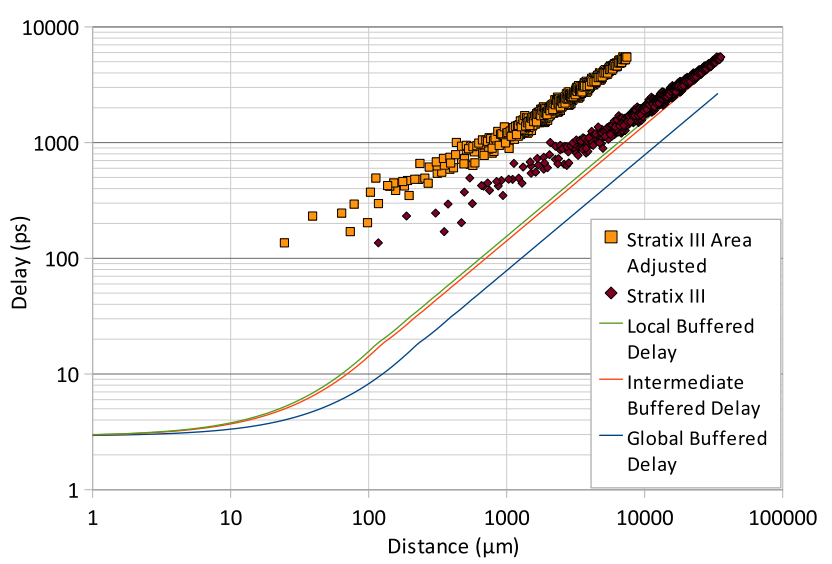

Figure 9: Point-to-Point Routing Delay

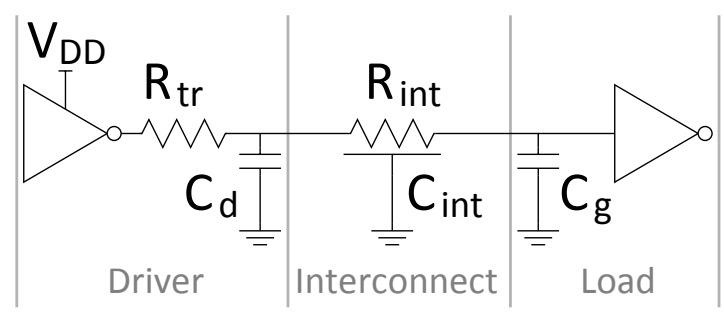

Figure 10: RC Model of Wire Delay

$(2 \times)$ to CMOS for the same length of wire.

Routing delays are more meaningful when "distance" is normalized to the amount of "logic" that can be reached. To approximate this metric, we adjust the FPGA routing distance by the square-root of the FPGA's overall area overhead vs. custom CMOS $(\sqrt{23 \times}=4.8 \times)$. Short local FPGA wires $(100 \mu \mathrm{m})$ have a logic density-normalized delay ratio $20 \times$, while long global wires $(7500 \mu \mathrm{m})$ have a delay ratio of only $9 \times$. The short wire delay ratio is comparable to the overall delay overhead for full processors, but the long wire delay ratio is half that, suggesting that FPGAs are less affected by long wire delays than custom CMOS.

\subsection{Off-Chip Memory}

Table 12 gives a brief overview of off-chip DRAM latency and bandwidth as commonly used in processor systems. Random read latency is measured on Intel DDR2 and DDR3 systems with off-chip (65 ns) and on-die (55 ns) memory controllers. FPGA memory latency is the sum of the memory controller latency and closed-page DRAM access time. While these estimates do not account for real access patterns, they are enough to show that off-chip latency and throughput ratios between custom CMOS and FPGA are far lower than for in-core circuits like complete processors.

\subsection{Summary of Building Block Circuits}

A summary of our estimates for the FPGA vs. custom CMOS delay and area ratios can be found in Table 13 . The range of delay ratios (8-75×) is smaller than the range of area ratios $(2-210 \times)$. Multiplexers have the highest delay ratios and hard blocks only have a small impact on delay ratios. Hard blocks are particularly area-efficient (SRAM, adders, multipliers), while multiplexers and CAMs are particularly area-inefficient.

In previous work, Kuon and Rose reported an average of $3.0-3.5 \times$ delay ratio and $18-35 \times$ area ratio for FPGA vs. standard cell ASIC for a set of complete circuits [4]. Al-

\begin{tabular}{lccc}
\hline & $\begin{array}{c}\text { Custom } \\
\text { CMOS }\end{array}$ & FPGA [53] & Ratio \\
\hline DDR2 Frequency (MHz) & 533 & 400 & 1.3 \\
DDR3 Frequency (MHz) & 800 & 533 & 1.5 \\
Read Latency (ns) & $55-65$ & $100-133$ & 2 \\
\hline
\end{tabular}

Table 12: Off-Chip DRAM Latency and Throughput. Latency assumes closed-page random accesses.

\begin{tabular}{lcc}
\hline Design & Delay Ratio & Area Ratio \\
\hline Processor Cores & $18-26$ & $17-27$ \\
\hline SRAM 1rw & $7-10$ & $2-5$ \\
SRAM 4r2w LUTs / LVT & $13 / 10$ & $143 / 23$ \\
CAM & 14 & $100-210$ \\
Multiplier & $17-22$ & $4.5-7.0$ \\
Adder & $15-20$ & $4.5-7.0$ \\
Multiplexer & $20-75$ & $>100$ \\
Pipeline latch & $12-19$ & - \\
Routing & $9-20$ & - \\
Off-Chip Memory & $1.3-2$ & - \\
\hline
\end{tabular}

Table 13: Delay and Area Ratio Summary

though we expect both ratios to be higher when comparing FPGA against custom CMOS, our processor core delay ratios are higher but area ratios are slightly lower, likely due to custom processors being optimized more for delay at the expense of area compared to typical standard cell circuits.

\section{IMPACT ON MICROARCHITECTURE}

The area and delay differences between circuit types and substrates measured in Section 4 affects the microarchitectural design of circuits targeting custom CMOS vs. targeting FPGAs. As FPGA designs often have logic utilization (area) as a primary design constraint, we expect that area ratios will have more impact on microarchitecture than delay ratios.

\subsection{Pipeline Depth}

Pipeline depth is one of the fundamental choices in the design of a processor microarchitecture. Increasing pipeline depth results in higher clock speeds, but with diminishing returns due to pipeline latch delays. The analysis by Hartstein et al. shows that the optimal processor pipeline depth for performance is proportional to $\sqrt{\frac{t_{p}}{t_{o}}}$, where $t_{p}$ is the total logic delay of the processor pipeline, and $t_{o}$ is the delay overhead of a pipeline latch [49].

Section 4.8 showed that the delay ratio of registers (i.e., $t_{o}$ FPGA vs. $t_{o}$ custom CMOS, $\sim 15 \times$ ) on FPGAs is lower than that of a complete processor (approximately $t_{p}$ FPGA vs. $t_{p}$ custom CMOS, $\sim 22 \times$ ), increasing $t_{p} / t_{o}$ on FPGA. The change in $t_{p} / t_{o}$ is roughly $(22 / 15)$, suggesting soft processors should have pipeline depths roughly $20 \%$ longer compared to an equivalent microarchitecture implemented in custom CMOS. Today's soft processors prefer short pipelines [54] because soft processors are simple and have low $t_{p}$, and not due to a property of the FPGA substrate.

\subsection{Partitioning of Structures}

The portion of a chip that can be reached in a single clock cycle is decreasing with each newer process, while transistor 
switching speeds continue to improve. This leads to microarchitectures that partition large structures into smaller ones and partition the design into clusters or multiple cores to avoid global communication [7].

In Section 4.9, we observed that after adjustment for the reduced logic density of FPGAs, long wires have a delay ratio roughly half that of a processor core. The relatively faster long wires lessen the impact of global communication, reducing the need for aggressive partitioning of designs for FPGAs. In practice, FPGA processors have less logic complexity than high-performance custom processors, further reducing the need to partition.

\subsection{ALUs and Bypassing}

Multiplexers consume much more area on FPGAs than custom CMOS (Section 4.7), making bypass networks that shuffle operands between functional units more expensive on FPGAs. The functional units themselves are often composed of adders and multipliers and have a lower $4.5-7 \times$ area ratio. The high cost of multiplexers reduces the area benefit of using multiplexers to share these functional units.

There are processor microarchitecture techniques that reduce the size of operand-shuffling networks relative to the number of ALUs. "Fused" ALUs that perform two or more dependent operations at a time increase the amount of computation relative to operand shuffling, such as the common fused multiply-accumulate unit and interlock collapsing ALUs $[55,56]$. Other proposals cluster instructions together to reduce the communication of operand values to instructions outside the group $[57,58]$. These techniques may benefit soft processors even more than hard processors.

\subsection{Cache Organization}

Set-associative caches have two common implementation styles. Low associativity caches replicate the cache tag RAM and access them in parallel, while high associativity caches store tags in CAMs. High associativity caches are more expensive on FPGAs because of the high area cost of CAMs (100-210 $\times$ bit density ratio). In addition, custom CMOS caches built from tag CAM and data RAM blocks can have the CAM's decoded match lines directly drive the RAM's word lines, while an FPGA CAM must produce encoded outputs that are then decoded by the SRAM, adding a redundant encode-decode operation that was not included in the circuits in Section 4.4. In comparison, custom CMOS CAMs have minimal delay and $2-3 \times$ area overhead compared to RAM allowing for high-associativity caches to have an amortized area overhead of around $10 \%$, with minimal change in delay compared to set-associative caches [59].

CAM-based high-associativity caches are not area efficient in FPGA soft processors and soft processor caches should have lower associativity than similar hard processors. Soft processor caches should also be higher capacity than similar hard processors because of the area efficiency of FPGA SRAMs (2-5× density ratio).

\subsection{Memory System Design}

The lower area cost of block RAM encourages the use of larger caches, reducing cache miss rates and lowering the demand for off-chip bandwidth to DRAM main memory. The lower clock speeds of FPGA circuits further reduce offchip bandwidth demand. The latency and bandwidth of off-chip memory is only slightly worse on FPGAs than on custom CMOS processors.
Low off-chip memory system demands suggest that more resources should be dedicated to improving the performance of the processor core than improving memory bandwidth or tolerating latency. Techniques used to improve the memory system in hard processors include DRAM access scheduling, non-blocking caches, prefetching, memory dependence speculation, and out of order memory accesses.

\subsection{Out-of-Order Microarchitecture}

Section 4.1 suggests that processor complexity does not have a strong correlation with area ratio, so out-of-order microarchitectures seem to be a reasonable method for improving soft processor performance. There are several styles of microarchitectures commonly used to implement precise interrupt support in pipelined or out-of-order processors and many variations are used in modern processors [60,61]. The main variations between the microarchitecture styles concern the organization of the reorder buffer, register renaming logic, register file, and instruction scheduler and whether each component uses RAM- or CAM-based implementations.

FPGA RAMs have particularly low area cost (Section 4.2), but CAMs are area expensive (Section 4.4). These characteristics favour microarchitecture styles that minimize the use of CAM-like structures. Reorder buffers, register renaming logic, and register files are commonly implemented without CAMs. There are CAM-free instruction scheduler techniques that are not widely implemented [6,62], but may become more favourable in soft processors.

If a traditional CAM-based scheduler is used in a soft processor, its capacity would tend to be smaller than on hard processors due to area, but the delay ratio of CAMs $(15 \times)$ is not particularly poor. Reducing scheduler area can be done by reducing the number of entries or the amount of storage required per entry. Schedulers can be data-capturing where operand values are captured and stored in the scheduler, or non-data-capturing where the scheduler tracks only the availability of operands, with values fetched from the register file or bypass networks when an instruction is finally issued. Non-data-capturing schedulers reduce the amount of data that must be stored in each entry of a scheduler.

On FPGAs, block RAMs come in a limited selection of sizes, with the smallest commonly being $4.5 \mathrm{kbit}$ to $18 \mathrm{kbit}$. Reorder buffers and register files are usually even smaller but are limited by port width or port count so processors on FPGAs can have larger capacity ROBs, register files, and other port-limited RAM structures at little extra cost. In contrast, expensive CAMs limit soft processors to small scheduling windows (instruction scheduler size). Microarchitectures that address this particular problem of large instruction windows with small scheduling windows may be useful in soft processors [63].

\section{CONCLUSIONS}

We have presented area and delay comparisons of processors and their building block circuits implemented on custom CMOS and FPGA substrates. In $65 \mathrm{~nm}$ processes, we find FPGA implementations of processor cores have 18$26 \times$ greater delay and $17-27 \times$ greater area usage than the same processors implemented using custom CMOS. We find that the FPGA vs. custom delay ratios of most processor building block circuits fall within the delay ratio range for complete processor cores, but that area ratios vary more. Building block circuits such as adders and SRAMs that have dedicated hardware support on FPGAs are particularly 
area-efficient, while multiplexers and CAMs are particularly area-inefficient. The measurements' precision is limited by the availability of custom CMOS design examples in the literature. The measurements can also change as both CMOS technology and FPGA architectures evolve and may lead to different design choices.

We have discussed how our measurements impact microarchitecture design choices: Differences in the FPGA substrate encourage soft processors to have larger, low-associativity caches, deeper pipelines, and fewer bypass networks than similar hard processors. Also, out-of-order execution is a valid design option for soft processors, although scheduling windows should be kept small.

\section{REFERENCES}

[1] Altera. Nios II processor. http://www.altera.com/products/ip/processors/nios2/.

[2] Xilinx. MicroBlaze soft processor. http://www.xilinx.com/tools/microblaze.htm.

[3] ARM. Cortex-M1 processor. http://www.arm.com/products/processors/cortex-m/cortexm1.php.

[4] I. Kuon and J. Rose. Measuring the Gap Between FPGAs and ASICs. IEEE Trans. Computer-Aided Design of Integrated Circuits and Systems, 26(2), Feb. 2007.

[5] D. Chinnery and K. Keutzer. Closing the Gap Between ASIC \& Custom, Tools and Techniques for High-Performance ASIC Design. Kluwer Academic Publishers, 2002.

[6] S. Palacharla et al. Complexity-Effective Superscalar Processors. SIGARCH Comp. Arch. News, 25(2), 1997.

[7] V. Agarwal et al. Clock Rate versus IPC: The End of the Road for Conventional Microarchitectures. SIGARCH Comp. Arch. News, 28(2), 2000.

[8] P. Metzgen and D. Nancekievill. Multiplexer Restructuring for FPGA Implementation Cost Reduction. In Proc. DAC, 2005.

[9] P. Metzgen. A High Performance 32-bit ALU for Programmable Logic. In Proc. FPGA, 2004.

[10] P. H. Wang et al. Intel Atom Processor Core Made FPGA-Synthesizable. In Proc. FPGA, 2009.

[11] S.-L. Lu et al. An FPGA-based Pentium in a Complete Desktop System. In Proc. FPGA, 2007.

[12] S. Tyagi et al. An Advanced Low Power, High Performance, Strained Channel 65nm Technology. In Proc. IEDM, 2005.

[13] K. Mistry et al. A 45nm logic technology with high-k+metal gate transistors, strained silicon, $9 \mathrm{cu}$ interconnect layers, $193 \mathrm{~nm}$ dry patterning, and $100 \%$ pb-free packaging. In Proc. $193 n m$ dry patt
$I E D M, 2007$.

[14] P. Bai et al. A $65 \mathrm{~nm}$ Logic Technology Featuring $35 \mathrm{~nm}$ Gate Lengths, Enhanced Channel Strain, $8 \mathrm{Cu}$ Interconnect Layers, Low-k ILD and $0.57 \mu^{2}$ SRAM Cell. In Proc. IEDM, 2004.

[15] S.K.H. Fung et al. 65nm CMOS High Speed, General Purpose and Low Power Transistor Technology for High Volume Foundry Application. In Proc. VLSI, 2004.

[16] A. S. Leon et al. A Power-Efficient High-Throughput 32-Thread SPARC Processor. IEEE Journal of Solid-State Circuits, $42(1), 2007$

[17] U.G. Nawathe et al. Implementation of an 8-Core, 64-Thread, Power-Efficient SPARC Server on a Chip. IEEE Journal of Solid-State Circuits, 43(1), 2008.

[18] G. Gerosa et al. A Sub-2 W Low Power IA Processor for Mobile Internet Devices in $45 \mathrm{~nm}$ High-k Metal Gate CMOS. IEEE Journal of Solid-State Circuits, 44(1), 2009.

[19] G. Schelle et al. Intel Nehalem Processor Core Made FPGA Synthesizable. In Proc. FPGA, 2010.

[20] R. Kumar and G. Hinton. A Family of 45nm IA Processors. In Proc. ISSCC, 2009

[21] Sun Microsystems. OpenSPARC. http://www.opensparc.net/.

[22] J. Davis et al. A 5.6GHz 64kB Dual-Read Data Cache for the POWER6 Processor. In Proc. ISSCC, 2006.

[23] M. Khellah et al. A $4.2 \mathrm{GHz} 0.3 \mathrm{~mm}^{2} 256 \mathrm{~kb}$ Dual-V ${ }_{c c}$ SRAM Building Block in 65nm CMOS. In Proc. ISSCC, 2006.

[24] P. Bai. Foils from "A 65nm Logic Technology Featuring 35nm Gate Lengths, Enhanced Channel Strain, $8 \mathrm{Cu}$ Interconnect Layers, Low-k ILD and $0.570 .57 \mu \mathrm{m}^{2}$ SRAM Cell". IEDM, 2004.

[25] L. Chang et al. A 5.3GHz 8T-SRAM with Operation Down to $0.41 \mathrm{~V}$ in $65 \mathrm{~nm}$ CMOS. In Proc. VLSI, 2007.

[26] S. Hsu et al. An $8.8 \mathrm{GHz} 198 \mathrm{~mW} 16 \times 64 \mathrm{~b} 1 \mathrm{R} / 1 \mathrm{~W}$ Variation-Tolerant Register File in 65nm CMOS. In Proc. ISSCC, 2006.

[27] S. Thoziyoor et al. CACTI 5.1. Technical report, HP Laboratories, Palo Alto, 2008.
[28] C. E. LaForest and J. G. Steffan. Efficient Multi-Ported Memories for FPGAs. In Proc. FPGA, 2010.

[29] K. Pagiamtzis and A. Sheikholeslami. Content-Addressable Memory (CAM) Circuits and Architectures: A Tutorial and Survey. IEEE Journal of Solid-State Circuits, 2006.

[30] K. McLaughlin et al. Exploring CAM Design For Network Processing Using FPGA Technology. In Proc. AICT-ICIW, 2006

[31] J.-L. Brelet and L. Gopalakrishnan. Using Virtex-II Block RAM for High Performance Read/Write CAMs. http://www.xilinx.com/support/documentation/ application_notes/xapp260.pdf, 2002.

[32] I. Arsovski and R. Wistort. Self-Referenced Sense Amplifier for Across-Chip-Variation Immune Sensing in High-Performance Content-Addressable Memories. In Proc. CICC, 2006.

[33] D. W. Plass and Y. H. Chan. IBM POWER6 SRAM Arrays. IBM Journal of Research and Development, 51(6), 2007.

[34] W. Hu et al. Godson-3: A Scalable Multicore RISC Processor with x86 Emulation. IEEE Micro, 29(2), 2009.

[35] A. Agarwal et al. A Dual-Supply $4 \mathrm{GHz} 13 \mathrm{fJ} / \mathrm{bit} / \mathrm{search}$ $64 \times 128 \mathrm{~b}$ CAM in $65 \mathrm{~nm}$ CMOS. In Proc. ESSCIRC 32, 2006

[36] S.K. Hsu et al. A 110 GOPS/W 16-bit Multiplier and Reconfigurable PLA Loop in 90-nm CMOS. IEEE Journal of Solid-State Circuits, 41(1), 2006.

[37] W. Belluomini et al. An 8GHz Floating-Point Multiply. In Proc. ISSCC, 2005

[38] J.B. Kuang et al. The Design and Implementation of Double-Precision Multiplier in a First-Generation CELL Processor. In Proc. ICIDT, 2005.

[39] P. Jamieson and J. Rose. Mapping Multiplexers onto Hard Multipliers in FPGAs. In IEEE-NEWCAS, 2005

[40] A. Agah et al. Tertiary-Tree 12-GHz 32-bit Adder in $65 \mathrm{~nm}$ Technology. In Proc. ISCAS, 2007.

[41] S. Kao et al. A 240ps 64b Carry-Lookahead Adder in 90nm CMOS. In Proc. ISSCC, 2006.

[42] S. B. Wijeratne et al. A 9-GHz 65-nm Intel Pentium 4 Processor Integer Execution Unit. IEEE Journal of Solid-State Circuits, 42(1), 2007.

[43] X. Y. Zhang et al. A 270ps 20mW 108-bit End-around Carry Adder for Multiply-Add Fused Floating Point Unit. Signal Processing Systems, 58(2), 2010.

[44] K. Vitoroulis and A.J. Al-Khalili. Performance of Parallel Prefix Adders Implemented with FPGA Technology. In Proc. NEWCAS Workshop, 2007.

[45] M. Alioto and G. Palumbo. Interconnect-Aware Design of Fast Large Fan-In CMOS Multiplexers. IEEE Trans. Circuits and Systems II, 2007.

[46] Altera. Stratix III Device Handbook Volume 1. 2009.

[47] D. Lewis et al. The Stratix II Logic and Routing Architecture. In Proc. FPGA, 2005.

[48] E. Sprangle and D. Carmean. Increasing Processor Performance by Implementing Deeper Pipelines. SIGARCH Comp. Arch. News, 30(2), 2002.

[49] A. Hartstein and T. R. Puzak. The Optimum Pipeline Depth for a Microprocessor. SIGARCH Comp. Arch. News, 30(2), 2002

[50] M. S. Hrishikesh et al. The Optimal Logic Depth per Pipeline Stage is 6 to 8 FO4 Inverter Delays. In Proc. ISCA 29, 2002.

[51] H.B. Bakoglu and J.D. Meindl. Optimal Interconnection Circuits for VLSI. IEEE Trans. Electron Devices, 32(5), 1985.

[52] International Technology Roadmap for Semiconductors. http://www.itrs.net/Links/2007ITRS/Home2007.htm, 2007.

[53] Altera. External Memory Interface Handbook, Volume 3. 2010.

[54] Peter Yiannacouras et al. The Microarchitecture of FPGA-based Soft Processors. In Proc. CASES, 2005.

[55] N. Malik et al. Interlock Collapsing ALU for Increased Instruction-Level Parallelism. SIGMICRO Newsl., 23(1-2), 1992

[56] J. Phillips and S. Vassiliadis. High-Performance 3-1 Interlock Collapsing ALU's. IEEE Trans. Computers, 43(3), 1994.

[57] P. G. Sassone and D. S. Wills. Dynamic Strands: Collapsing Speculative Dependence Chains for Reducing Pipeline Communication. In Proc. MICRO 37, 2004

[58] A. W. Bracy. Mini-Graph Processing. PhD thesis, University of Pennsylvania, 2008

[59] M. Zhang and K. Asanovic. Highly-Associative Caches for Low-Power Processors. In Kool Chips Workshop, Micro-33, 2000.

[60] J.E. Smith and A.R. Pleszkun. Implementing Precise Interrupts in Pipelined Processors. IEEE Trans. Computers, 37(5), 1988.

[61] G.S. Sohi. Instruction Issue Logic for High-Performance, Interruptible, Multiple Functional Unit, Pipelined Computers. IEEE Trans. Computers, 39:349-359, 1990.

[62] F. J. Mesa-Martínez et al. SEED: Scalable, Efficient Enforcement of Dependences. In Proc. PACT, 2006.

[63] M. P. et al. A Decoupled KILO-Instruction Processor. Proc. $H P C A, 2006$ 